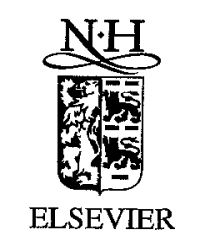

Surface Science 377-379 (1997) 355-359

\title{
Coherent plasmon-surface phonon oscillations in a GaAs Schottky barrier sample
}

\author{
W. de Jong ${ }^{\text {a }}$, A.F. van Etteger ${ }^{\text {a }}$, P.J. van Hall ${ }^{\text {b }}$, Th. Rasing ${ }^{\text {a,* }}$ \\ ${ }^{a}$ Research Institute for Materials, Nijmegen, The Netherlands \\ ${ }^{b}$ Department of Physics, Eindhoven University of Technology, Eindhoven, The Netherlands
}

Received 1 August 1996; accepted for publication 15 October 1996

\begin{abstract}
Excitation of a biased GaAs-Au Schottky barrier sample by intense fs laser pulses leads to coherent oscillations that are probed by time-resolved second harmonic generation experiments. The observed oscillations agree very well with the results of MC simulations and appear to be plasma oscillations modified by surface phonons.
\end{abstract}

Keywords: Carrier dynamics; GaAs; Schottky barrier; Second harmonic generation

\section{Introduction}

Coherent oscillations such as Bloch oscillations and coherent phonons have been the subject of extensive studies in recent years [1-3]. Coherent oscillations on the femtosecond time-scale are important due to promising applications as a source of coherent $\mathrm{THz}$ radiation [4]. Here we report, to our knowlcdge for the first time, the direct temporal observation of coherent plasmaphonon oscillations in the depletion region of a $\mathrm{Au}-\mathrm{GaAs}$ Schottky barrier (SB) system. These coupled modes have recently been observed on clean GaAs surfaces but they were not found in oxide-capped surfaces [5]. Bare plasma oscillations have been observed in bulk semiconductors [2].

In the band-bending region of a SB a very high density of charge carriers can be created by excita-

\footnotetext{
* Corresponding author. Fax: +31 243652190 ; e-mail: theoras@sci.kun.nl
}

tion with very intense ultrashort laser pulses. Due to the existing d.c. electric field the electrons and holes are accelerated in opposite directions and thus a counteracting field is built up, causing a collapse of the net electric field. The charge carriers attain a finite velocity and due to their inertia the counteracting field becomes bigger than the initially applied field. If the scattering and the damping forces are not too big, coherent oscillations of the electron-hole plasma and thus of the electric field can occur. This electric field dynamics at the SB interface can be sensitively probed by timeresolved electric field induced optical second harmonic generation (SHG), due to its intrinsic interface sensitivity [6].

Both the slow (1-10 ps) and the fast $(<200 \mathrm{fs})$ components of the observed time dependence can be very well described by ensemble Monte Carlo simulations. Whereas the slow component is rclated to the rccovery of the collapsed clectric field and is governed by an effective RC time 
constant, the observed $\mathrm{THz}$ oscillations can be shown to originate from coupled plasma-interface phonon modes.

\section{Experimental}

The Schottky barrier sample we used for our experiments was grown on an $\mathrm{n}^{+} \operatorname{GaAs}(100)$ substrate. The actual barrier is formed by a 3000 A-thick n-type GaAs (doping concentration: $10^{17} \mathrm{~cm}^{-3}$ ) layer and a semi-transparant $100 \AA$ thick $\mathrm{Au}$ film to allow for laser excitation through the metal top contact. The experiments were performed at room temperature and with a reverse bias voltage of $3 \mathrm{~V}$ applied to the sample.

For the SHG measurements we used a modelocked titanium sapphire laser operating at $\lambda=$ $846 \mathrm{~nm}$ to produce trains of $45 \mathrm{fs}$ pulses at a $82 \mathrm{MHz}$ repetition rate. The photon energy at this wavelength is $1.466 \mathrm{eV}$, which is about $46 \mathrm{meV}$ above the band-gap of GaAs.

The SHG contributions from the bulk GaAs and from the interface can be separated by looking at the azimuthal anisotropy of the SHG signal [7]. To detect the $\mathrm{THz}$ oscillations, time-resolved pump probe experiments were performed at a fixed rotation angle of the sample. Pump and probe beams were generated by a beam splitter. The probe pulses were delayed with respect to the pump pulses by a computer-controlled delay stage with $0.1 \mu \mathrm{m}$ step size. The probe beam was p-polarized and the p-component of the generated specular SHG intensity was detected by a photon counting system. The probe and pump beams also generate a sum frequency signal, corresponding to the crosscorrelation of the pump and probe pulses, which enables us to control the spatial and temporal overlap of the pulses and to determine the pulse width. The pump-probe measurements were performed for different laser powers in the pump beam. The average laser power in the probe beam is kept constant at about $2 \mathrm{~mW}$.

Most measurements are performed with the pump beam s-polarized (perpendicular to the polarization of the probe) and with the (011) axis of the sample parallel to the plane of incidence. In this case the electric field-independent bulk signal is maximal and, due to a cross term between the large bulk and the smaller electric field-dependent interface contribution, a relatively large electric field-dependent signal can also be measured. This is important to cnable measuring oscillations in the electric field. At this sample orientation the change in the SHG intensity is directly proportional to the change in the electric field at the interface.

\section{Results}

After excitation of the carriers by a not toostrong pump pulse $\left(\sim 20 \mathrm{~W} / \mathrm{cm}^{2}\right)$ the observed SHG collapses on a timescale of several picoseconds, due to the collapse of the electric field at the interface. The signal recovers after several tens to hundreds of picoseconds, depending on the excitation intensity and size of the excitation laser spot $[8,9]$.

When the excitation laser power is further increased, the collapse becomes faster and the recovery becomes slower. At these high laser powers a clear oscillatory-like structure develops on the collapsing tail, as can be seen in Fig. 1. This figure shows the measured SHG intensity for an average pump intensity of $125 \mathrm{~W} / \mathrm{cm}^{2}$ at the sample. The final value of 900 counts per second consists mainly of the electric field-independent

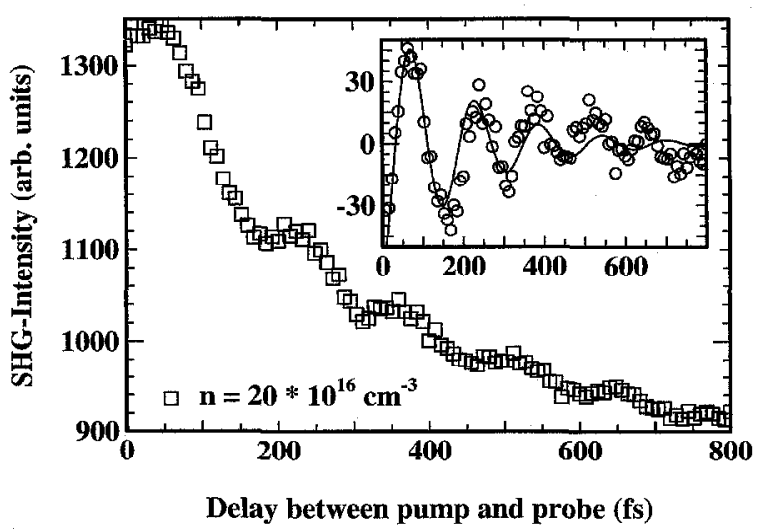

Fig. 1. Measured time evolution of the second harmonic intenstity for an average pump intensity of $125 \mathrm{~W} / \mathrm{cm}^{2}$ at the sample. The inset shows the data-points with an exponential background subtracted. The solid line is a single frequency fit. 
(and thus time-independent) bulk contribution to the SHG Intensity $[7,8]$. The inset of the figure shows the same data points but now with the exponential background subtracted. The solid line is an exponentially decaying sine fit with a single frequency.

\section{Simulations}

To compare the observations with theory, ensemble Monte Carlo simulations are performed for these experiments. In these simulations the device is modelled schematically with a parabolic band bending. The $0.3 \mu \mathrm{m}$-thick GaAs layer is separated from the Au by the Schottky barrier. Due to its heavy n-type doping the superlattice is assumed to form no barrier for the electrons and a large one for the holes. The applied bias voltage creates an interface charge at the Au film which is compensated for by a constant intrinsic space charge throughout the whole depletion layer, giving rise to a linear decreasing electric field and a parabolic potential. This means that in this model the depletion width is constant and equal to the GaAs layer thickness, whereas the doping concentration is varied with the applied bias voltage. In an actual Schottky device, the doping level is constant and the depletion width changes with bias voltage [10]. Although this model is rather schematic, we believe it nevertheless contains the basic physics needed for describing semi-quantitatively the experimental results for high reverse bias voltages $(3.0 \mathrm{~V})$, when the depletion region approaches the GaAs layer thickness. The carriers are created in the depletion layer according to the absorption coefficient of GaAs for the incident laser light. The subsequent transport and energy relaxation of both electrons and holes in the GaAs layer are computed in a Monte Carlo simulation [11], accounting for the various scattering mechanisms such as optical and acoustic phonons, ionised impurity, intervalley, and carrier-carrier scattering $[12,13]$. Both electron-heavy-hole and electron-light-hole pairs are considered, including hole inter-band transitions [14] and the Pauli exclusion principle. Standard $10^{5}$ electron-hole pairs were used. To calculate the net electric field after excitation of the charge carriers, the Poisson equation is solved in one dimension every $1 \mathrm{fs}$ of the calculation, resulting in a time-dependent local driving field in addition to the applied field. The simulation results for the high input powers show that shortly after excitation the net electric field starts oscillating. The inset of Fig. 2 shows the calculated electric field versus time after excitation and versus the position in the space-charge region at an excited density of carriers of $2 \times 10^{17} \mathrm{~cm}^{-3}$. The SB interface is at $0 \mathrm{~nm}$. In the middle of the space charge region the electric field is inverted within $200 \mathrm{fs}$ and starts oscillating. The curve at the bottom of Fig. 2 shows the time evolution of the net electric field as calculated at a depth of $9 \mathrm{~nm}$ in the semiconductor. Comparison of this curve with Fig. 1 shows that there is a very good agreement between the measurements and the simulations for this position. The calculated amplitude and dephasing of the oscillations are of the same order as those observed in the experiment.

At the interface the oscillations are damped, as can be seen in Hig. 2. This interface damping can

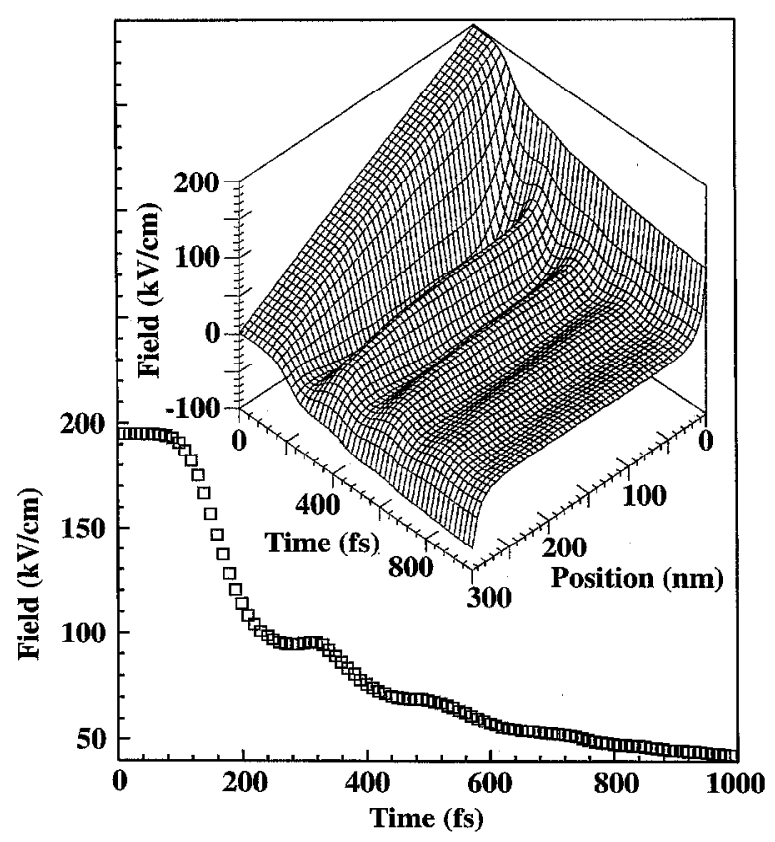

Fig. 2. Calculated time evolution of the electric field in the depletion region. The curve at the bottom is at a depth of $9 \mathrm{~nm}$ below the interface. 
be understood as follows. Very shortly after excitation a number of holes will reach the top contact, where these holes neutralize the metal surface charge, leading to a reduced field strength. If the photo-excited charge density significantly excecds the intrinsic one, the applied field may be destroyed totally by only a small part of the excited holes. It will take some time the field to be restored, that is for the illuminated part of the device to be recharged. Obviously, this recharging time is connected with the RC time constant of the device studied, which is basically the illuminated part on which a surplus of charge is present. In order to investigate the consequences for the carrier sweepout of such a recharging time, we introduced a so-called regeneration time constant $\tau_{\text {reg }}$ by which charge is transferred to the illuminated part of the top contact tending to keep the total voltage across the depletion layer constant. In the regime of low excitation intensities the measured results for the dependence on the spot size and the excitation intensity agree very well with the MC calculations [8].

\section{Discussion}

Fourier analysis of the measurements shows that the oscillations consist of more than one frequency component. The main frequency of the $125 \mathrm{~W} /$ $\mathrm{cm}^{2}$ curve is $6.3 \mathrm{THz}$ which corresponds to an energy of $26.1 \mathrm{meV}$; the second frequency with a smaller amplitude is $9.8 \mathrm{THz}(40.6 \mathrm{meV})$. The main frequency for the measured $45 \mathrm{~W} / \mathrm{cm}^{2}$ curve is $5.8 \mathrm{THz}(24.0 \mathrm{meV})$. The dependence of this main frequency on the density of excited carriers is much smaller than the square root dependence on the density of excited carriers for bare plasma oscillations. This deviation is due to coupling of the plasma frequency to the (power-independent) frequency of a phonon [15]. Using dispersion formulas [15] the expected frequencies can be calculated for given values of the phonon frequencies and the density of excited carriers. From these calculations it follows that the $9.8 \mathrm{THz}$ frequency is a coupling between the plasmon and the wellknown bulk LO phonon at $8.8 \mathrm{THz}(36.7 \mathrm{meV})$. The $5.8 \mathrm{THz}$ and $6.3 \mathrm{THz}$ frequencies are a coupling between the plasmon and a surface phonon at a frequency of about $7.8 \mathrm{THz}$ $(32.3 \mathrm{meV})$. This phonon frequency is lower than that of the bulk phonons. Phonons with a frequency around this value are also observed on clean GaAs surfaces but they were not found in oxide-capped surfaces [5].

\section{Conclusion}

The agreement between the experimental results and the simulations leads to the conclusion that the observed THz oscillations in the SHG Intensity are indeed directly measurable oscillations in the electric field at the Schottky barrier interface caused by plasma oscillations. The observed power dependence and the appearance of a second frequency are caused by coupling of the plasma oscillations with surface and bulk phonons.

\section{Acknowledgements}

We would like to thank W.C. van der Vleuten, C.M. van Es and P.A.M. Nouwens from the group of prof. dr. J.H. Wolter at the Eindhoven University of Technology for the preparation of the samples and Y.M. Chang and H.W.K. Tom for illuminating discussions. Part of this work was supported by the Stichting voor Fundamenteel Onderzoek der Materie (FOM), which is financially supported by the Nederlandse Organisatie voor Wetenschappelijk Onderzoek (NWO).

\section{References}

[1] G.C. Cho, W. Kütt and H. Kurz, Phys. Rev. Lett. 65 (1990) 764.

[2] W. Sha, Arthur L. Smirl and W.F. Tseng, Phys. Rev. Lett. 74 (1995) 4273.

[3] W. Fischler, P. Buchberger, R.A. Höpfel and G. Zandler, Appl. Phys. Lett. 68 (1996) 2778.

[4] H.G. Roskos, M.C. Nuss, J. Shah, K. Leo, D.A.B. Miller, A.M. Fox, S. Schmitt-Rink and K. Köhler, Phys. Rev. Lett. 68 (1992) 2216.

[5] Y.M. Chang, L. Xu and H.W.K. Tom, Ultrafast Phenomena X (Springer, Berlin, 1996), to be published. 
[6] Th. Rasing, Appl. Phys. A 59 (1994) 531, and references therein.

[7] W. de Jong, A.F. van Etteger, C.A. van't Hof, P.J. van Hall and Th. Rasing, Surf. Sci. 331-333 (1995) 1372.

[8] W. de Jong, A.F. van Etteger, C.A. van't Hof, P.J. van Hall and Th. Rasing, in: Ultrafast Processes in Spectroscopy, Eds. O. Svelto, S. De Silvestri and G. Denardo (Plenum, New York, 1996), to be published.

[9] P.C.M. Christianen, P.J. van Hall, H.J.A. Bluyssen and J.H. Wolter, Semicond. Sci. Technol. 9 (1994) 707.

[10] S.M. S7e, Physics of Semiconductor Devices, 2nd ed. (Wiley, New York, 1981).
[11] C. Jacoboni and L. Reggiani, Rev. Mod. Phys. 55 (1983) 645.

[12] C. Jacoboni and P. Lugli, in: The Monte Carlo Method for Semiconductor Device Simulation (Springer, New York, 1989).

[13] Hot Electron Transport in Semiconductors, Ed. L. Reggiani (Springer, New York, 1985).

[14] M. Costato and L. Reggiani, Phys. Status Solidi (b) 58 (1973) 461.

[15] T. Inaoka, Surf. Sci. 357/358 (1996) 87. 